\title{
PARTICIPAÇÃO DA ENFERMEIRA NO SERVIÇO DE AMBULATÓRIO E SAÚDE PÚBLICA DO HOSPITAL DAS CLÍNICAS DA FACULDADE DE MEDICINA DE RIBEIRÃO PRETO DA UNIVERSIDADE DE SÃO PAULO
}

\author{
Dulce Maria Vendrúsculo* \\ Gilda Cintra** \\ Guiomar Cácamo*** \\ M. ${ }^{2}$ Helena P. de Oliveira***
}

\begin{abstract}
RBEn/04
VENDRÚSCULO, D.M., colaboradores - Participação da enfermeira no serviço de ambulatório e saúde pública do Hospital das Clínicas da Faculdade de Medicina de Ribeirão Preto da Universidade de São Paulo. Rev. Bras. Enf.; RJ, $28: 28-34,1975$.
\end{abstract}

\section{I - INTRODUÇÃO}

As atividades do ambulatório do Hospital das Clínicas da Faculdade de Medicina de Ribeirão Preto tiveram início em 1957, visando ao atendimento a pacientes de Clínica Médica, Cirurgia, Obstetrícia e Pediatria.

A partir de 1963, quando o Serviço passou a contar com duas enfermeiras, estas se responsabilizaram pelo planejamento e definição das funções do pessoal de enfermagem.

O serviço de Triagem Médica, inicialmente ligado ao setor de Pediatria, estendeu-se a outras áreas médicas, facilitando assim, não só o atendimento aos doentes como a divisão de serviço de atendimento no ambulatório, em várias áreas de especialidade médica.
Em março de 1968, com a mudança do ambulatório para uma área específica, foram reorganizadas suas atividades, em termos de planejamento de programa de saúde, rotinas de atendimento e reestruturação das diversas clínicas de especialidades médicas.

As atividades de enfermagem de saúde pública foram iniciadas em julho de 1956, por instrutores da Escola de Enfermagem em colaboração com o Departamento de Medicina Social, sendo relacionadas com os objetivos desse Departamento.

\section{II - HISTÓRICO}

Este relatório pretende mostrar os

* Instrutora de Enfermagem Pediátrica da Escola de Enfermagem de Ribeirão Preto.

** Enfermeira do Ambulatório Geral do Hospital das Clínicas da Faculdade de Medecina de Ribeirão Preto, USP.

*** Enfermeira de Saúde Pública do Hospital das Clínicas da Faculdade de Medicina de Ribeirão Preto, USP. 
VENDRÚSCULO, D.M., colaboradores - Participação da enfermeira no serviço de am.bulatório e saúde pública do Hospital das Clínicas da Faculdade de Medicina de Ribeirão Preto da Universidade de São Paulo. Rev. Bras. Enf.; RJ, 28 : 28-34, 1975.

trabalhos que vimos realizando no setor de saúde pública do Hospital das Clínicas de Ribeirão Preto que inclue o ambulatório e Escola de Enfermagem. Em se tratando de um serviço dedicado à assistência à comunidade, é necessário aqui uma análise prévia das condições que Ribeirão Preto oferece, em termos de assistência médica hospitalar à sua população.

Ribeirão Preto, cidade situada ao norte do Estado de São Paulo, conta com uma população urbana de 195.680 habitantes e uma população rural de 16.620 habitantes, segundo o recenseamento de dezembro de 1970. Na assistência hospitalar, conta com 13 estabelecimentos, dos quais dois são governamentais; Hospital Santa Teresa (psiquiátrico) e o outro é o Hospital das Clínicas da Faculdade de Medicina, totalizando 2.934 leitos para o atendimento a diversas áreas e especialidades médicas.

Dada a sua localização geográfica, para esta cidade convergem pacientes de outras localidades, aumentando assim consideravelmente o número de pessoas que procuram nossos servicos, não só hospitalares como de ambulatório.

O setor de enfermagem de saúde pública, pertencente à Divisão de Enfermagem do Hospital das Clínicas da Faculdade de Medicina de Ribeirão Preto é parte integrante do atendimento dinâmico dessa população, não só nos aspectos preventivos como também curativos e educacionais. Neste setor a enfermeira tem uma participação mais direta na execução de programas de trabalho, visando a promoção de saúde do indivíduo e da comunidade.

\section{III — AMBULATORIO GERAL}

1. - OBJETIVOS GERAIS:

1.1 - Estabelecer diagnóstico e tratamento nas melhores condições técnicas possíveis.
1.2 - Selecionar pacientes que necessitam de internação.

1.3 - Servir de campo de pesquisas das moléstias que mais incidem sobre a comunidade.

1.4 - Servir de campo de ensino para médicos, estudantes de medicina, enfermagem, serviço social e outros profissionais.

\section{2. - OBJETIVOS GERAIS DO SER- VIÇO DE ENFERMAGEM}

2.1 - Prestar cuidados direto e indireto ao paciente.

2.2 - Fazer educação sanitária dos pacientes.

2.3 - Auxiliar em pesquisa e fornecimento de dados estatísticos ao Serviço de Estatística.

2.4 - Preparar e organizar o campo de estágio para alunos da Faculdade de Medicina, Enfermagem e outros profissionais.

\section{3. - AREAS DE ATENDIMENTO E} DIVERSOS ESPECIALIDADES MÉDICAS

- Clínica Médica e especialidades, abrangendo: dermatologia, cardiologia e endocrinologia.

- Ortopedia

- Ginecologia e Obstetrícia

- Neurologia e Neurocirurgia

- Cirurgias e Especialidades

- Pediatria e Especialidades

- Puericultura

- Clínica de Complexo Primário

As diversas clínicas e especialidades médicas atendem no ambulatório, obedecendo a programações já estabelecidas, bem como o horário e as rotinas de serviços pré-fixadas. 
VENDRÚSCULO, D.M., colaboradores - Participação da enfermeira no serviço de ambulatório e saúde pública do Hospital das Clínicas da Faculdade de Medicina de Ribeirão Preto da Universidade de São Paulo. Rev. Bras. Enf.; RJ, 28 : 28-34, 1975.

4, - ÁREA FÍSICA DE ATENDIMENTO AMBULATORIAL

O ambulatório geral abrange uma área de $1080 \mathrm{~m}^{2}$, subdivido em 4 alas laterais e uma central, comprendendo:

- Salas de atendimento médico: 28

- Ensino e orientação de paciente: 2

- Sala de curativo: 1

- Colheita de material para exames bacteriológicos (adultos e crianças): 1

- Sala de amostra de medicamento: 1

- Pesagem de pediatria: 1

- Sala de serviço: 1

- Balcão de recepção com a finalidade de receber os pacientes para o atendimento médico.

- Local de espera para consulta médica

- Sala de serviço de Enfermagem do ambulatório

- Sanitários para funcionários, médicos e pacientes: 16

\section{5 - ATIVIDADES DESENVOLVIDAS PELO SERVIÇO DE ENFERMAGEM:}

5.1 - Atividades administrativas: administração, organização, coordenação e supervisão do ambulatório geral.

5.2 - Programa de Educação Sanitária em ambulatório de diabetes: O presente trabalho vem sendo realizado desde 1969, contando com a colaboração da Equipe de Enfermagem, Departamento de Clínica Médica e com a participação direta da enfermeira responsável pelo setor de ambulatório.

\subsection{1 - Objetivos:}

- obter participação ativa do paciente diabético e de sua família no programa de educação e controle da doença.

- orientar o paciente e sua família na adaptação e introdução de novos hábitos em face da doença.

- orientar o paciente quanto a sua doença, etiologia, controle e cuidados físicos.
5.2.2 - Atividades desenvolvidas:

- elaboração de um plano de trabalho específico para o diabético.

- realização de discussão com a participação de pacientes.

- orientação individual após consulta médica.

- realização de aulas práticas com demonstrações - visando o preparo de material para a aplicação de insulina, dosagem e manuseio de seringa própria.

- distribuição de panfletos educativos e de cartão de identificação do paciente diabético.

5.3 - Centro de prevenção, detenção e tratamento do câncer ginecológico: Este trabalho foi iniciado em novembro de 1971, através do Departamento de Ginecologia, Obstetrícia e Pediatria da Faculdade de Medicina de Ribeirão Preto, e, colaboração do Serviço de Enfermagem do Ambulatório Geral.

5.3.1 - Atividades iniciais e programação:

- Ampliação da sala de colposcopia

- Mudança na unidade de atendimento de ginecologia e obstetrícia

- Elaboração de rotina de atendimento dos pacientes

- Reunião com a equipe de trabalho responsável

- Treinamento de funcionários para o atendimento na sala de colposcopia

- Treinamento de funcionários para a coleta de dados estatísticos e encaminhamento dos pacientes.

5.3.2 - Atividades de Enfermagem:

- Seguimento do paciente durante o exame

- Orientação do paciente ao exame a ser submetido.

- Controle do material colhido e encaminhamento do mesmo.

Nota: De novembro de 1971 a março de 1972 foram submetidos ao exame colposcópico 1.504 pacientes, sendo que destes, 
VENDRÚSCULO, D.M., colaboradores - Participação da enfermeira no serviço de ambulatório e saúde pública do Hospital das Clínicas da Faculdade de Medicina de Ribeirāo Preto da Universidade de São Paulo. Rev. Bras. Enf.; RJ, 28 : 28-34, 1975.

18 pacientes apresentaram resultados positivos.

5.4 - Tratamento Quimioterápico

5.5 - Profilaxia e tratamento da febre reumática

IV - ATIVIDADES DESENVOLVIDAS PELO SERVIÇO DE ENFERMAGEM DE SAÚDE PÚBLICA

1 - Objetivos Gerais do Setor de Saúde Pública

As atividades de Enfermagem de Saúde Pública foram iniciadas em julho de 1956, por instrutoras da Escola de Enfermagem de Ribeirão Preto, em colaboração com o Departamento de Medicina Social, tendo como finalidade principal o desenvolvimento e manutenção de um campo de ensino para estudantes de Enfermagem e Medicina.

Inicialmente os trabalhos basearamse em:

- Organização do Serviço de Imunização

- Atendimento de Ambulatório

- Controle de pacientes portadores de Moléstia de Chagas e seguimento da família através de visita domiciliária.

Esse serviço estendeu-se rapidamente, penetrando em outras áreas como $\mathrm{Ma}$ terno-Infantil e controle de moléstias transmissiveis (Sífilis e Tuberculose). Começou também a participar de atividades externas, atuando na comunidade através de programas educacionais, ministrando cursos de Educação e Saúde.

Os objetivos atuais do programa de Enfermagem de Saúde Pública baseiamse nos mesmos princípios estabelecidos desde o início de seu trabalho, dando mais ênfase, portanto, à Higiene Materno-Infantil, à Profilaxia das Doenças Transmissíveis, à Educação Sanitária em ambulatório e grupos de comunidade, promovendo e mantendo campo de ensino e pesquisa para as escolas.

2 - Atividades desenvolvidas

2.1 - Atividades Administrativas:
Organização, coordenação e supervisão das atividades de Saúde Pública.

2.2 - Ambulatório de Higiene Materno-Infantil:

- Ambulatório de Pré-Natal: Todas as gestantes registradas no ambulatório do Hospital das Clínicas de Ribeirão Preto, para controle de pré-natal, passam pela Enfermagem de Saúde Pública, a fim de receberem orientação sistemática quanto a importância do exame pré-natal, aos aspectos de gravidez, do parto e dos primeiros cuidados do recém-nascido. Este primeiro contato com a paciente é individual, onde a enfermeira se dispõe e esclarecer outros problemas apresentados pela gestante. ( $\mathrm{Na}$ primeira entrevista é preenchido uma ficha de identificação, possibilitando assim um controle mais rigoroso do comparecimento das pacientes às consultas médicas).

No retorno, as gestantes recebem orientação em grupo, tipo palestra, onde são abordados vários assuntos de interesse das pacientes.

As visitas domiciliárias se fazem quando necessárias, dando prioridade aos grupos de primigestas e nos casos que requerem maior cuidado.

As gestantes após orientação da enfermeira são encaminhadas à Sala de Imunização onde recebem vacina antitetânica.

Nota: As gestantes que apresentam Reação de Wassermann positivo são transferidas para o ambulatório de Medicina Social onde são controladas juntamente com os familiares.

2.3 - Ambulatório de Puericultura: Teve início em agosto de 1969, o atendimento feito pela Enfermagem de Saúde Pública.

2.3.1 - Consulta de Enfermagem no $10 .^{\circ}$ dia de vida: Todas as crianças nascidas no Hospital das Clínicas, residentes em Ribeirão Preto (com exceção das que pertencem aos bairros de Vila Lo- 
VENDRÚSCULO, D.M., colaboradores - Participação da enfermeira no serviço de ambulatório e saúde pública do Hospital das Clínicas da Faculdade de Medicina de Ribeirão Preto da Universidade de São Paulo. Rev. Bras. Enf.; RJ, 28 : 28-34, 1975.

bato, Vila Tibério, Vila Virgínia e Ipiranga), no $100^{\circ}$ dia de vida passam no Amulatório e são atendidas pela Enfermagem de Saúde Pública, a fim de que as mães recebam orientação de enfermagem sistemática, quanto ao aspecto de crescimento e desenvolvimento, cuidados gerais com a criança, etc.

Nota: Vide modelo anexo do roteiro de consulta de Enfermagem.

2.3.2 - Puericultura em Geral: Todas as crianças, após consulta médica, passam pela Enfermagem de Saúde Pública, para que as mães das mesmas possam ser orientadas quanto a importância dos cuidados para uma saúde perfeita.

O grupo etário de prioridade é o de zero a dois anos de idade.

Recebem orientação quanto a:

- Alimentação em geral

- Higiene Pessoal

- Imunização

- Profilaxia de Verminoses

2.4 - No Ambulatório de Padiatria Geral: A assistência infantil de Enfermagem tem como finalidade principal a orientação às mães sobre cuidados na manutenção da saúde de seus filhos dando ênfase no problema de:

- Alimentação em Geral

- Cuidados Higiênicos em Geral

- Profilaxia e tratamento de Verminoses

- Imunização em Geral

2.5 - Ambulatório de Complexo Primário: Em agosto de 1970 foi reorganizado o atendimento (médico-pediatria e enfermagem de saúde pública) do Ambulatório de Complexo Primário do Hospital das Clínicas de Ribeirão Preto.

Finalidade: Todas as crianças de zero a 11 anos de idade, registradas no Ambulatório de Pediatria, com diagnóstico de Complexo Primário, deverão após consulta médica, passar pela Enfermagem de Saúde Pública para investigação do foco, orientação de enfermagem, encaminhamento dos familiares e fornecimento de medicação.

Atividades desenvolvidas:

a) Entrevista com a família - No primeiro contato da enfermeira com o paciente é preenchida uma folha de orientação juntamente com uma ficha que serve para controle dos casos.

b) Visita domiciliária para pesquisa do foco: Todos os casos diagnosticados, residentes em Ribeirão Preto recebem a visita da enfermeira de Saúde Pública, cuja finalidade é:

- pesquisa do foco de contágio

- orientação quanto à necessidade dos familiares se submeterem ao exame e tratamento

- verificação da existência de pessoas doentes do pulmão - na família ou vizinhança

- encaminhamento dos adultos em contato com a criança para exame de abreugrafia no dispensário de tuberculose

Os casos são visitados quantas vezes forem necessárias até que seja descoberto o foco de contágio. Após as consultas médicas as crianças passam pela Enfermagem onde as mães recebem orientação em geral quanto aos cuidados da manutenção de Saúde. Educação Sanitária dos familiares, fornecimento de medicação e controle dos retornos.

\section{6 - Ambulatório de sífilis}

Finalidades: Pacientes com "Lues" independentes da Clínica a que pertencem são encaminhados à Enfermagem de Saúde Pública com pedido de interconsulta para o ambulatório de Medicina Social.

Na Saúde Pública o paciente é submemetido a uma entrevista cujo objetivo é:

- descoberta dos casos novos e contatos

- controle do tratamento

- educação do paciente e familiares em relação aos aspectos da doença. 
VENDRÚSCULO, D.M., colaboradores - Participação da enfermeira no serviço de ambulatório e saúde pública do Hospital das Clínicas da Faculdade de Medicina de Ribeirão Preto da Universidade de São Paulo. Rev. Bras. Enf.; RJ, $28: 28-34,1975$.

Além destes citados a enfermeira dá uma explicação da doença e sua evolução natural, modo de transmissão e importância da descoberta do contato.

Os casos de Sífilis residentes em Ribeirão Preto são visitados pela Enfermeira.

2.7 - Ambulatório do Departamento de Medicina Social

Sala de Imunização: A Enfermagem de Saúde Pública, supervisiona e orienta toda a imunização ativa e passiva feita nos pacientes do Hospital das Clínicas de Ribeirão Preto.

a) Imunização ativa artificial - todos os pacientes, principalmente dos ambulatórios de Pediatria, Obstetrícia e Medicina Social passam pelo Serviço de Imunização, onde são orientados quanto a vacinas, reações possíveis e importância de comparecer nos retornos para completar o esquema.

A Enfermagem de Saúde Pública colabora com a Campanha de Imunização Ativa na Comunidade e Instituiçōes.

b) Imunização passiva artificial todos os pacientes que necessitam de imunização passiva (soros) são encaminhados a Sala de Imunização, onde recebem o soro, juntamente com as devidas orientações.

c) Outras atividades são executadas como:

— Teste de sensibilidade à Penicilina

- Teste de suscetibilidade à Difteria (Teste de Schick)

- Aplicação de auto-vacina

- Prova Tuberculínica, reação de Mantoux (Old Tuberculin) para os pacientes de Ambulatório, de Enfermaria e outros que procuram o nosso Serviço:

- Aplicação de Injeções em geral
2.8 - Sala de Colheita de Sangue para Pesquisa de "Doença de Chagas e Lues":

E rotina do Hospital das Clínicas todos os pacientes registrados passarem pela Sala onde é feito o exame de sangue como meio de controle.

\section{9 - Serviço de Abreugrafia}

Todos os pacientes no Hospital das Clínicas fazem abreugrafia como rotina médica, com exceção das gestante e crianças de zero a quatorze anos.

\subsection{0 - Serviços Prestados à Comuni-} dade

Quando se faz necessário, a Enfermagem de Saúde Pública faz visitas domiciliares para: pesquisa de foco (Tuberculose e Sífilis), assistência de Enfermagem nos casos que requerem maiores cuidados, gestantes e imunização em Instituições.

O Serviço de Enfermagem de Saúde Pública colabora com a Divisão Regional de Saúde de Ribeirão Preto.

CONCLUSÃO: Embora existindo programação específica em certas áreas prioritárias, as necessidades de atuação de uma unidade de Saúde Pública integrado a um Hospital Geral abrangem aspectos, os mais variados, que deverão ser observados pela equipe de Saúde Hospitalar e o pessoal que atua no campo de Saúde Pública e Ambulatório.

O reconhecimento das normas de serviço e atividades que poderão ser desenvolvidas por estes dois setores é de primorcial importância para que haja uma integração uniforme e contínua no planejamento e cuidados executados no Hospital e posteriormente através do acompanhamento ambulatorial ou domicilar pelas enfermeiras de saúde Pública. 
VENDRÚSCULO, D.M., colaboradores - Participação da enfermeira no serviço de ambulatório e saúde pública do Hospital das Clínicas da Faculdade de Medicina de Ribeirão Preto da Universidade de São Paulo. Rev. Bras. Enf.; RJ, 28 : 28-34, 1975.

\section{BIBLIOGRAFIA}

1 - Carvalho, J.F. - Papel da Enfermeira. Trabalho apresentado no II Seminário sobre Educação - Ribeirão Preto, 1969.

2 - Ciconelli, M.I. et al. - Papéis e Funções dos Enfermeiros do Hospital das Clínicas da Faculdade de Medicina de Ribeirão Preto da Universidade de São Paulo. Trabalho apresentado no II Seminário sobre Educação - Ribeirão Preto, 1969.

3 - Costa, J. \& Almeida, M.C.P. - Atividades desenvolvidas pelo Serviço de Enfermagem de Saúde Pública do Hospital das Clínicas da Fa- culdade de Medicina de Ribeirão Preto da Universidade de São Paulo - Relatório apresentado no II Seminário sobre Educação Ribeirão Preto, 1969.

4 - Costa, J. - Aproveitamento dos Ambulatórios como Areas de Ensino Ensino Prático de Enfermagem. Rev. Bras. Enfermagem, 22 (1) (2): 19-25, jan./jun. 1970 .

5 - Hospital das Clínicas da Faculdade de Medicina de Ribeirão Preto Serviço de Enfermagem de Saúde Pública - Relatório anual qualitativo e quantitativo das atividades. 